\title{
Multiple game based rehabilitation platform instead of intensified conventional balance training in patients with stroke: pilot study
}

Imre Cikajlo ( $\boldsymbol{D}$ imre.cikajlo@ir-rs.si )

University rehabilitation institute https://orcid.org/0000-0002-8428-7621

Marko Rudolf

University rehabilitation institute

Renato Mainetti

Universita degli Studi di Milano

Nunzio Alberto Borghese

Universita degli Studi di Milano

Research article

Keywords: Balance, rehabilitation, virtual reality, posture, center of pressure, exercise intensity, exergaming

Posted Date: May 14th, 2019

DOI: https://doi.org/10.21203/rs.2.9581/v1

License: (c) This work is licensed under a Creative Commons Attribution 4.0 International License.

Read Full License 


\section{Abstract}

Background: People who survive a stroke usually suffer for moderate to severe movement disorders such as uncontrollable movements of the limb, posture and balance disorders. An intensive and repetitive physiotherapy and balance training are often a key to functional restoration of movements. Recently modern rehabilitation centers have offered virtual reality supported balance training with exergames in addition to the conventional therapy. However, the choice of the appropriate exergame is still not straightforward. Methods: We designed a randomized pilot study investigating how different types of balance training in addition to the physiotherapy program influence on functional outcomes. 20 eligible participants were randomized into 2 groups; exergaming vs conventional balance training. Multiple-game platform with exergames supporting single leg exercises, weight shifting, balancing and standing up and equivalent conventional physiotherapy exercises were used. Center of pressure was monitored during exergaming and clinical impact was evaluated with the Four Square Step Test (FSST), Timed Up \& Go, $10 \mathrm{~m}$ Walk Test (10MWT), Romberg, Sharpened Romberg, Clinical Test for Sensory Interaction in Balance in both groups. Statistical tests were used to find statistically significant $(p<0.05)$ differences and Cohen's U3 test to examine the effect sizes. Results: Participants of both groups demonstrated substantively and statistically significant improvements of functional balance, in particular the exergaming group (FSST $p=0.009, \mathrm{U} 3=0.9$ and 10MWT $p=0.008, \mathrm{U} 3=0.9$ ). . However, significant differences between the groups were found only in tests with eyes closed, Sharpened Romberg test $(p=$ $0.05)$ and standing on the right leg $(p=0.035)$. We found a decrease of center of pressure area for up to $20 \%$ in the exergaming group. Conclusions: In the exergaming group the participants achieved improved functional balance that could be well targeted by the selection of games and objectively evaluated. The modern approach had a great potential by choosing the appropriate game to target the exercise, but the low statistical power may hinder the equivalence of both additional balance training methods. However, the exergaming provided independent balance training, also feasible without physiotherapist's strenuous work that may be crucial for future home or telerehabilitation services.

\section{Background}

\subsection{Motor function rehabilitation after stroke}

Cerebrovascular accident or stroke is a consequence of blood supply interruption in some parts of the brain due to the blood spill or blood block in the blood vessel by a clot. People having a stroke are affected cognitively and physically and deprived of daily living functions. If the stroke is very severe, the person may die immediately, if not, consequences depends on which part of the brain is injured and how severe was the stroke. However, nearly $80 \%$ of survivors of nearly 15 million strokes worldwide each year (WHO) suffer for motor function impairment and are in need for comprehensive motor rehabilitation to get back to their everyday life routine (1). For successful motor rehabilitation an intensive exercises are required (2) that is often limited due to time constraints and lack of therapists even in the top rehabilitation clinics. A temporary solution is to prepare the patients for an additional home exercise, which is in most cases unsuccessful due to the lack of motivation. Those patients rarely meet the 
prescribed home rehabilitation regimens (3) and are advised to visit the outpatient clinic. Clinical studies suggested at least 3 hours of timed exercises per week (4). The authors agreed that duration and intensity of exercises would have been crucial for optimal rehabilitation outcomes, no matter if rehabilitation was carried out in clinical or home environment.

Both rehabilitation environments, clinical and home, have one crucial element in common, i.e. patient's motivation. Several researchers have developed rehabilitation games, also in virtual reality, to increase the motivation of the patients and engage them in the motor rehabilitation more intensively (5). The main focus of rehabilitation games may be attracting patients' attention and motivating them to achieve higher score, while the virtual reality based rehabilitation offers interactive simulated environments. The users are engaged in these environments that could be similar to the real world or imaginary objects and events. The rehabilitation games are often controlled by sensors, recently contactless motion tracking sensors (Microsoft Kinect, Leapmotion, etc) or force plates (Wii Balance Board, AMTI, etc) that serve also as an assessment devices. Patients with severe stroke may often need a passive or active rehabilitation robot providing upper limb (6), (7) or lower limb support (8), (9), (10).

\subsection{Exergaming in balance training}

Balance and posture are considered complex motor and cognitive functions depending on various factors, such as muscle power, mobility, and sensory information from musculoskeletal system, visual and vestibular systems. Cognitive and emotional factors should also not be neglected (11).

Cerebrovascular accident or stroke often affects balance, posture and mobility, increased risk of falls (1) and limited daily living activities. Clinical findings emphasize the importance of large number of regular repetitions of functional movements to achieve neuroplastic changes in the brain, which would result in functional improvement of motor functions (2), (3). However, the conventional physiotherapy is often limited in providing large number of repetitions due to the limited time available for each patient, limited number of skilled professionals or other related organizational problems (12), (4). In last decade researchers have proposed exergaming and virtual reality (VR) supported rehabilitation as a tool to increase repeatability and controllability of the physiotherapeutic processes.

The literature review provides a long list of publications emphasizing the importance and advantages of using VR and exergames in the rehabilitation. The VR and exergames provide variety of options for eliciting the maximum voluntary activity of the patient, like accurate task repetitions, frequency, intensity, changes of virtual environments and gradual increase of task complexity (13). Nevertheless, the patients can experience practicing simulated daily activities (e.g. crossing the road) even without the continuous supervision of the therapist. This may increase the intensity without a need for additional therapists. Laver et al (14) carried out an extensive review and came to conclusions that using VR in patients with cerebrovascular accident can be an effective rehabilitation method, especially as an addition to the 
conventional physiotherapy. The approach can prolong the time available for therapy and improve day-today performance comparing with the standard treatment in upper limb daily activities of patients with stroke. Corbetta et al (15) reported that substitution of specific part of the conventional rehabilitation with VR can elicit improvements in gait, balance and mobility in person with stroke. However, most of the reports highlighted that further clinical evidences would be required to justify the superiority of any approach.

Rehabilitation Wayout in Responsive Home Environments (REWIRE) was a European project involving more than 10 partners to enable patients who have completed inpatient rehabilitation in rehabilitation centers to continue with the physiotherapy at home (16). Demanding commercial video games have been considered too complex for most patients, thus the researchers in cooperation with the physiotherapists have developed rehabilitation game system based on Gentile's Motor Skills Taxonomy. The rehabilitation games were based on the theme "Living on the Farm", providing a calm and relaxing atmosphere for patients. The user participated in the game as an avatar. The system enabled five difficulty levels and provided visual and audio notifications about the correct posture. Microsoft Kinect was used as motion capture device and Wii Balance Board for ground reaction forces monitoring. The data assessed were stored locally and transmitted to the server to enable the physiotherapist a remote insight into the patient's performance and required changes of the telerehabilitation program.

\subsection{Objectives of the paper}

Previous studies demonstrated that implementing rehabilitation games into the process of physiotherapy can be not only effective or provide good clinical experience, but also scientific studies provided clinically evidences (15). Most of the studies were intended for the controlled clinical settings and the entire process was carried out by the medical professional, often a physiotherapist. The objective of our paper was to demonstrate that using specific rehabilitation games based on Gentile's taxonomy for additional balance training could be as successful as conventional physiotherapy and would allow targeting specific neuromotor control exercises. The physiotherapist was present only to supervise the process, ensure safety for the patient and to prevent any event that could potentially lead to an accident. We expect that motivation and independent use of exergames may in the future contribute to the prolonged rehabilitation at patient's home, while the therapist will remain as a consultant. Hereby we raise the following research hypothesis:

- Additional targeted multi-exergaming program can be equivalent to the intensive balance training in the conventional physiotherapy

- Selected exergames based on motor skill taxonomy would target the specific balance disorder

Full clinical trial with 4-week program would answer the first hypothesis entirely, but a pilot randomized clinical trial with smaller number of participants and five sessions demonstrated that applied exergaming instead of additional strenuous balance training can provide an objective assessment and training tool. 


\section{Methods}

\subsection{Rehabilitation system}

The rehabilitation system consisted of a larger LCD screen (42"), a computer, a Microsoft Kinect ${ }^{\mathrm{TM}}$ camera (RGB camera has a standard resolution of $640 \times 480$ at 30 FPS up to $1024^{\star} 768$ at 12 FPS.), Wii Balance Board (Nintendo Inc., Redmond, WA, USA) and $6 \mathrm{~m} 2$ of dedicated space. The Microsoft Kinect ${ }^{\mathrm{TM}}$ camera was mounted above or under the LCD to cover the entire space dedicated for exergamming. The participant was standing in front of the LCD at the distance of about 2-2.5m on the Wii Balance Board (WBB) (Figure 1). The feet and the legs were held apart as at normal upright posture. After the start of the REWIRE platform, the system had been configured and the participant/physiotherapist was able to choose a game and set up a difficulty level and duration time.

The games were designed to follow the modified Gentile's taxonomy of motor skill (17). We chose three games suitable for balance, posture and weight shift according to the taxonomy, i.e.: 3A-4A body stability without object manipulation and $3 \mathrm{C}-4 \mathrm{C}$ body transport without object manipulation.

The Animal Hurdler game (Figure 2): The aims of the exergame were balance and posture, weight shift and single leg standing. The participant had to step over some small creatures that approach the virtual participant (avatar) by rising the foot. The duration was set to $3 \mathrm{~min}$ and the difficulty level was set to 3 defining the required height for rising legs (distance of the foot from the ground).

The Fruit Catcher game (Figure 2): This exergame was targeted at balance and posture, weight shift and steps. The goal of the game was to catch fruits and avoid chocolate eggs falling from the top of the tree. The virtual participant stood below the tree with a basket on the head and moved the body laterally to catch the fruits falling from the tree. The participant scored after the fruit had fallen into the basket. The fruits fell from different positions on the branches in the horizontal axis. The duration was set to $3 \mathrm{~min}$ and the difficulty level was set to 3 defining the distances for stepping or weight shifting (distance of the fruit from the center).

The Horse Runner game (Figure 2): The aims of this exergame were balance and posture, and muscles strengthening by sit-to-stand exercise. The virtual participant sat on top of the horse that was running in the woods. The horse progressed forward automatically, determining the pace of the exergame. By doing squats the participant could slow down or speed up the horse and avoid hitting branches with the avatar's head; standing up would make the horse run faster and the participant would get bonuses (collect floating honey jars). The duration was set to $3 \mathrm{~min}$ and the difficulty level was set to 3 defining the number of repetition per minute. 
For the study 30 acute and subacute stroke survivors were recruited, but only 20 were eligible (Figure 3 ). They met the inclusion criteria defined by the responsible physician: 1. first ischemic or hemorrhagic stroke, 2. first admission to rehabilitation programs, 3. ability to follow instructions and participate - Mini Mental State Examination (MMSE) $>=25$, 4. ability to walk independently - minimal FIM® score of 5 . A physiotherapist randomized the recruited patients into two groups by drawing lots:

- 10 patients in the conventional physiotherapy (control) group ; 9 males and 1 female, $51.8 \pm 15.5$ years old, 4 had left side and 6 had right side affected after ischemic (6) or hemorrhagic (4) stroke (7.4 months prior to participation in the study).

- 10 patients in the exergaming group; 6 males and 4 female, $50.3 \pm 7.9$ years old, 5 had left side and 4 had right side affected and 1 had both sides affected after ischemic (9) or hemorrhagic (1) stroke (4 months prior to participation in the study).

Patients were tested with the MMSE, a brief screening test that assesses the severity of cognitive impairment and has a maximum score of 30 . The mean MMSE score in the control group was $27.6 \pm 1.8$ and in the gaming group $28.6 \pm 1.3$. Additionally all patients were tested with FIM® instrument (FIM) estimating the level of the patient's disability and indicating how much assistance was required for the individual to carry out the activities of daily living. All admitted patients had FIM score 6 .

\subsection{Research protocol}

All participants in the study were part of the same neurotherapeutic treatment at the rehabilitation center. The study comprised 5 sessions of intensive physiotherapy in 5 consecutive days in two groups. Both groups received additional therapy; one group taking a conventional physiotherapy, and the other exergaming physiotherapy. One day prior to the study and a day after the study balance and motor abilities of the participants were tested with validated clinical tests.

The gaming group received regular neurotherapeutic treatment and additional daily $15 \mathrm{~min}$. of goal based gaming with three different games supported by the REWIRE system. Each participant played the weight shifting game Fruit catcher for $3 \mathrm{~min}$ at the level 3 , then took a rest for $3 \mathrm{~min}$ and continued with the squat exercise game Horse runner for $3 \mathrm{~min}$ at the level 3 . The participant took a rest for $3 \mathrm{~min}$ before continuing with additional 3 min of one leg standing exercise with Animal hurdler game, also at the level 3. The participant was standing on the WBB during the exercising, while the movements of the entire body were recorded with MS Kinect camera (Figure 1). If any support or help in terms of physical or cognitive assistance was needed, the physiotherapist would have provided assistance.

The control group also received regular neurotherapeutic treatment and additional daily $15 \mathrm{~min}$. of intensive exercises. The manual exercises for the control group were selected specifically to functionally match the exercises performed with exergames. These exercises consisted of weight shifting to left and right leg, squats, lifting legs and balancing at wall bars. The amount of exercises was equivalent to the 
exergaming in the gaming group (3 times 3 min with 3 min of resting time). Each participant started with 3 min of weight shifting from left to right leg, keeping the position for $3 \mathrm{~s}$ and then shifting the weight to the left leg. The $3 \mathrm{~min}$ break followed by $3 \mathrm{~min}$ of squat workout. The last exercise after having a 3 min of rest was the alternate lifting of the lower limbs for $3 \mathrm{~min}$. The physiotherapist was present all the time and could offer physical or cognitive assistance, if needed.

\subsection{Data assessment}

\subsubsection{Center of pressure}

Center of pressure (COP) displacement was assessed by WBB that features 4 pressure sensors, one at each corner. The WBB measured 4 vertical forces when the participant was standing with feet apart on the board. The system computed the common COP displacement from provided force data and known board dimensions. The COP displacement data provided by WBB are perhaps not the most reliable and accurate and not preferable for clinical diagnostic (18), but its repeatability and acceptable accuracy is appropriate for exergaming. Besides, the WBB serves as a portable and inexpensive substitute for the force plate in home environments and telerehabilitation applications.

COP displacement area depended on the balance/sway performance of the participant. Balance disorders and instability resulted in wider and larger COP area (Figure 4). The COP area was estimated by the $95 \%$ confidence ellipse (19). The computation was done by subtracting the mean value (Mv) from the set of data (DS) and computing the scale factor ( $\mathrm{x}$ ) by chi-square inverse cumulative distribution function for the given probability (95\%):

Due to technical limitations, Equation 1 has been placed in the Supplementary Files section.

where $\mathrm{G}$ is the Gamma function $\mathrm{G}(\mathrm{n})=(\mathrm{n}-1)$ !. The covariance matrix (COV) of the mean centered variable (DS-Mv) comprised variances along the diagonal. The COV was scaled by factor $x$. Then we computed the eigenvector and eigenvalues of the matrix:

Due to technical limitations, Equation 2 has been placed in the Supplementary Files section.

Scaled eigenvectors were applied to project the ellipse (Figure 4) in the parametric form: 
Due to technical limitations, Equation 3 has been placed in the Supplementary Files section.

\subsubsection{Games score}

Total achieved points in each exergame depended on the chosen difficulty level, time elapsed and game score achieved. The difficulty level was set to 3 (medium speed) for all three games. The data were collected during 3 min exercise for each game and each session (day). The games score was not considered as a measure for participants' rehabilitation progress, but provided valuable information on participant's effort and cognitive understanding of the exercise. .

\subsubsection{Clinical tests}

Clinical tests were carried out a day prior to the first session and a day after the last session in all participants. Appropriate tests for balance, posture and mobility were selected: Four Step Square Test (FSST) to test dynamic balance at stepping over objects sideways, forward and backwards (20), Timed Up \& Go (TUG) to assess balance, mobility and fall risk (21) and $10 \mathrm{~m}$ walk test (MWT) to assess gait performance (22). Additionally Romberg's Test (ROM), sharpened Romberg's Test (sROM), standing on the left leg (STOLL), standing on the right leg (STORL) and the foam and dome test, also called the "Clinical Test for Sensory Interaction in Balance" (CTSIB) were carried out up to 45 s with eyes open and closed. Romberg's Tests with eyes open evaluated the vision, proprioception and vestibular sensory systems at balancing. The upper limit was set to $45 \mathrm{~s}$. Visual input was removed by closing the eyes and participants relied on vestibular and/or proprioceptive sensory systems only. They could not compensate, if there was any severe lesion. Therefore the clinical Romberg test may not detect vestibular problems accurately (23). The CTSIB was used to quantify the postural control in clinical terms under different conditions (24).

\subsection{Data analysis}

We examined the COP area in the gaming group during the exergame therapy, requiring adequate postural responses to maintain balance when doing exercises. It was expected that participants with poor balancing abilities would show evidently larger COP area. The COP area was examined in each individual for 5 sessions in 5 consecutive days. We divided the analysis into 3 parts according to the exergame used and calculated the mean COP area and its standard deviation for each session. On top of that we plot the regression curve/line. We also compared the COP area across the three exergames, expecting significant differences due to the different balance/postural tasks in each exergame; standing on one leg, weight shifting, standing up and balancing.

Matlab (MathWorks, Natick MA, USA) was used to extract raw data, calculate COP 95\% confidence ellipse and its area. Matlab Statistical Toolbox was used to calculate the mean; standard deviation of the COP area and repeated measures ANOVA with Tukey HSD posthoc test (SPSS 14, IBM statistics Inc., USA) was used to determine the differences between the exergames. Before the analysis data were checked for 
normality and equality of variances. Additionally the exergaming scores were statistically analyzed between the sessions for each exergame. The significance level was set to $p=0.05$.

We carried out a separate analysis of the performed clinical tests for both groups the control group and for the gaming group. Data obtained in both groups were tested for normality and equality of variances with Bartlett's test. In a case the homogeneity of variances test failed $\left(c^{2} p<0.05\right)$, we used Man-Whitney $\mathrm{U}$ Test to determine the differences between the interventions and Wilcoxon Test for time effect. If the data passed the homogeneity test, we would apply 2-way-ANOVA for intervention and time effect. The significance level was set to $p=0.05$. Cohen's U3 index (25) was used to find the effect sizes. The U3 defines the proportion of data in gaming group that were smaller than the median values of control group. There was no effect at U3 $=0.5$ and maximal at 0 when all data of gaming group were above the median of the control group or 1 when all data of the gaming group were below the median of the control group (effect size: small 0.4/0.6, medium 0.3/0.7, and large 0.2/0.8). Matlab Statistical Toolbox (MathWorks, Natick MA, USA) with Measures of Effect Size (MES) Toolbox (26) and GNU PSPP (Free Software Foundation, Inc., Boston MA, USA) were used for analysis.

\section{Results}

\subsection{Exergaming group}

All 10 participants diagnosed with stroke who were randomly assigned to the gaming group accomplished the 5 sessions according to the protocol and completed the assigned clinical tests. The COP analysis excluded missing data of one participant. However, the participant completed all clinical tests and accomplished the game therapy successfully, thus was not excluded from the study.

\subsubsection{Center of pressure area}

Results of the calculated COP area for each exergame per session are presented in Table 1. We removed the data of the participant nr. 7 (however, the participant finished the entire protocol) for the FruitCatcher game due to unreliable recordings. We calculated the COP area with the ellipse covering the $95 \%$ of the data assessed. Data were averaged over the participants for each exergame and session separately. The mean values and the standard deviation values are presented in the Table 1 . The decreasing regression line (Figure 5) demonstrated a potentially gradual decrease of COP area and thus smaller range of motion reflecting in the improved balance capabilities. The regression line in the Animal hurdler game was the steepest, indicating the greatest impact on the COP and dynamic balance.

The effects of different games on the COP area during balance training through the sessions were examined. Prior to the repeated measures analysis of variances (ANOVA) a Mauchly's test of sphericity 
(27) indicated that the assumption of sphericity in the data had been violated, $\chi 2=45.599, p<10^{-4}$. Therefore the Greenhouse-Geisser correction was applied to the degrees of freedom (df) for the Fdistribution $(e=0.456)$. The effects within subjects were calculated with the repeated measures ANOVA after the corrections had been made. Corrections had no effect on sum of squares or the $F$, but affected $\mathrm{df}$ and the p-value. We found the changes in COP area (Figure 5) thought the sessions statistically insignificant $(p=0.222)$. Additionally the interaction effect between time and the exergame was also found insignificant $(p=0.114)$. However, the Tukey HSD post-hoc test found statistically significant differences between the exergames AnimalHurdler and FruitCatcher, AnimalHurdler and HorseRider ( $p<$ 0.003). But statistically insignificant differences were found in the mean COP area between the exergames FruitCatcher and HorseRider $(p=0.088)$.

\subsubsection{Success in gaming}

The total points achieved in particular exergames were not comparable to each other. Comparison of total points achieved was carried out between the sessions for each particular exergame.

The overview of total points achieved in the AnimalHurdler shows that the participants successfully accomplished the game in the last 2 sessions with $26 \%$ higher score (Figure 6 ). The detailed analysis of the median and interquartile range demonstrated large variation of the results (the score range 100-3500, $p=0.306$ ), however, the median values showed higher score at the end () of the exergaming. In the $4^{\text {th }}$ session we have noticed sample skewness.

In the FruitCatcher exergame the participants achieved $8 \%, 10 \%$ higher mean score at the $4^{\text {th }}$ (mean 2438 points) and $5^{\text {th }}$ session (mean 2460 points, $p=0.458$ ), respectively. The analysis of the median values (Figure 6), the distribution of the score and interquartile range demonstrated that participants had been able to accomplish the game successfully with equal score even at the session 2 (mean 2316 point) and 3 (mean 2303 points). The outcomes of the FruitCatcher game for the session 1 were corrupt or missing for 3 participants, thus we considered the score for this session invalid.

On the other hand the results of the HorseRider exergame show that participants were able to achieve high score even at the session 1 (mean 3071 points) or at the session 4 (mean 3060 points). The median value of the total points dropped at the sessions 2 and 3 and the $1.5 \mathrm{x}$ interquartile range demonstrated a large variation in total game score, particularly in session 3. However, the mean values at HorseRider game slightly dropped at the session 2 (mean 2832 points) and was higher in the session 3 (mean 2945 points) and session 5 (mean 2990 points, $p=0.268$ ).

\subsubsection{Clinical outcomes}


The balance, postural and mobility tests FSST, TUG and MWT demonstrated functional improvements (Table 2); the FSST failed the Bartlett $\left(c^{2}=27.79\right)$ homogeneity of variances test and the Wilcoxon's test found significant functional improvement after the training (mean 13.21s vs 10.24s, $p=0.009, \mathrm{U}=0.9$ ). Additionally, the test found that 9 participants improved their FSST and only 1 did not. The positive changes of the 10 MWT test (mean 8.76s vs $\left.7.14 \mathrm{~s}, c^{2}=23.69\right)$ were found significant $(p=0.008, U 3=$ 0.9). Nine participants improved their MWT time, but the MWT did not improve neither was worse for 1 participant. The Wilcoxon's test was also used for TUG $\left(c^{2}=25.19\right)$ and despite 8 participants improved their TUG and 2 did not, the changes were not statistically significant (mean 9.56s vs 8.46s, $p=0.092$ ). However, the effect size was still rather large $(Z=-1.68$, U3 $=0.7)$.

CTSIB performance with eyes open improved after the training for 2 participants and remained the same for the rest and were not sensitive due to the ceiling effect in majority of participants (mean $38.83 \mathrm{~s} v \mathrm{~s}$ 43.52s; $c^{2}=$ Inf, $p=0.18$ ). In STOLL test with eyes closed (mean 1.61s vs $\left.4.62 s, c^{2}=14.12, p=0.093\right) 5$ participants improved their results, 3 did not and 2 achieved the same results as before the exergaming. Four participants were more successful in STORL with eyes closed, 4 were not and 2 participants achieved the same results as before the exergaming, although the changes were not statistically significant (mean $3.92 \mathrm{~s}$ vs $2.82 \mathrm{~s}, \mathrm{c}^{2}=29.62, \mathrm{p}=0.674$ ).

The outcomes of the ROM test (Table 2) with eyes open could be also neglected due to the ceiling effect of the majority of the participants (mean $44.33 \mathrm{~s}$ vs $43.75 \mathrm{~s}, \mathrm{c}^{2}=$ inf, $p=0.317$ ). However, the changes in ROM test with eyes closed (mean 36.79s vs 41.49s) were significant over time (ANOVA $p=0.046, c^{2}=$ $5.11)$, but there was no effect size due to the ceiling effect $(U 3=0.5)$. The sROM test with eyes closed indicated minor changes in time (19.65s vs $\left.18.96 \mathrm{~s}, \mathrm{p}=0.969, \mathrm{c}^{2}=2.14, \mathrm{U} 3=0.6\right)$. The sROM with eyes opened indicated successful training for 3 participants, while the other participants achieved the ceiling results (mean $34.56 \mathrm{~s}$ vs $42.11 \mathrm{~s}, \mathrm{p}=0.369, \mathrm{c}^{2}=6.53$ ).

\subsection{Conventional rehabilitation group}

All 10 participants diagnosed with stroke were randomly assigned to the control group and accomplished the additional exercises sessions according to the protocol and accomplished all clinical tests.

\subsubsection{Clinical outcomes}

The participants in the control group accomplished the TUG and MWT mobility and balance tests $(15.18 \mathrm{~s}$ vs $12.17 \mathrm{~s}$ and $12.34 \mathrm{~s}$ vs $9.82 \mathrm{~s}$ ) faster after the training and the results were confirmed statistically significant (Table 3 ) by Wilcoxon's test $\left(c^{2}=25.19, p=0.011\right.$ and $c^{2}=23.69, p=0.008$, respectively). Nine participants improved their MWT time. Eight participants improved their TUG time, 1 failed to do so and 1 
remained with the same result. However, the group mean time did not improve in FSST (12.75s vs 14.50s, $p=0.575)$.

On the CTSIB with eyes open (Table 3 ) the additional training had practically no effect (43.50s vs $45 \mathrm{~s}, \mathrm{p}=$ 0.317 , U3 $=0.5$ ) due to the ceiling effect of the majority of the participants. Larger effect was achieved with eyes closed (29.13s vs 34.77s, $p=0.341$, U3 =0.3). Positive but minor changes in STORL with eyes open and STOLL with closed eyes were not statistically significant (Table 3), $p=0.753, p=0.273$, respectively.

The ROM test with eyes closed demonstrated large effect size (26.46s vs 40.29s, U3 = 0.2) and significant changes in time ANOVA $\left.p=0.046, c^{2}=5.108\right)$. The sROM tests with eyes open and closed demonstrated minor changes within the control group (Table 3 ) and time effect was statistically insignificant $\left(p>0.369, c^{2}<6.53\right)$.

\subsection{Differences between the groups}

The differences in age, time since stroke, affected side and MMSE were statistically analyzed for 20 participants randomized into 2 groups. The variances were assumed equal (Leven's Test) for gender and time since stroke variables, therefore t-test assuming unequal variances was used. We found minor and statistically insignificant differences between the two groups of participants (Table 4).

Despite the gaming group demonstrated significant progress in motor and balance tests MWT $(p=0.008)$ and FSST ( $p=0.009$ ), but not also gait test TUG ( $p=0.092)$, the outcomes were not significantly different ( $p>0.05$, Man-Whitney U Test) from the control-group in any of these tests (Figure 7).

Standing on the right leg with eyes closed (STORL EC) demonstrated significant differences between the groups ( $p=0.035$, Man-Whitney U Test, Figure 8$)$, but medium effect size (U3 = 0.3).

Practically no statistically significant differences between the gaming group and control group were found in tests performed with eyes open (ROM EO, sROM EO, STOL(R)L EO and CTSIB EO). Mean values between the groups were found different in ROM EC and SROM EO tests, but differences in these tests between the gaming and control group were statistically insignificant (ANOVA group effect $p=0.206$, 0.319 , respectively). Statistically significant differences between the participants in gaming and control groups were found only in sROM $\left(p=0.05, c^{2}=2.15\right.$, ANOVA) and STORL $\left(p=0.035, c^{2}=29.63\right.$ ManWhitney U Test) tests, both performed with closed eyes.. These tests demonstrated also small to medium effect sizes, U3 $=0.4$ and 0.3 , respectively (Figure 8).

\section{Discussion}

\section{$1.1 \quad$ Exergaming group}


Participants in the gaming group managed to accomplish the exergames as required by the protocol. The mean COP area calculated from the anterior-posterior and mediolateral displacements have decreased from the $1^{\text {st }}$ session to the last session for the 3 applied exergames. The COP area was gradually decreasing from session to session in particular for the HorseRunner exergame. For the AnimalHurdler exergame the participants' COP area was rather alternating, though the regression line's negative slope showed a reduction of COP area size. The exergame FruitCatcher was evidently an easier game to play requiring only medio-lateral movements. The participants' mean COP area increased in the $2^{\text {nd }}$ session and then gradually decreased until the last session. However, the statistically significant difference of the mean COP area between the AnimalHurdler and HorseRunner suggests that these exergames targeted different gait, balance or posture parameters. While AnimalHurdler was intended for balance and single leg standing, the HorseRunner was designed for practicing balance and standing up (16). The FruitCatcher was designed for balance, posture and weight transfer, therefore the statistical differences in COP area displacements found with the AnimalHurdler exergame were not surprising. Nevertheless, the FruitCatcher and HorseRunner exergames required the most similar functional balance tasks as shown by the outcomes. These findings may be important at choosing the appropriate exergame when targeting specific balance or gait parameter. We found significant improvement of balance parameters in exergaming group with FSST, gait parameters with the 10MWT and positive changes with TUG mobility test, even if statistically non-significant. The participants were able to finish the FSST test that required stepping forward, sideway and backward on the squares in clockwise and counterclockwise directions, even faster than before the exergaming program. This means that they may have improved their dynamic balance performance as the test required loading on the affected side while facing forward. The COP also showed an improvement of participants' ability to shift the center of mass and maintain stability while stepping. These findings were in line with our expectations and previously published studies suggesting that the COP area displacement had been positively correlated to the balance and gait parameters (28). The studies also reported on results that were correlated to the muscle strength, gait performance, balance, postural control and stability and their improvement with the reduction of COP area after the rehabilitation/physiotherapy in patients diagnosed with stroke (29) and appeared to be significantly different in persons diagnosed with stroke and healthy persons (30).

The attractiveness of the exergames plays an important role in rehabilitation outcomes (31) therefore the achieved game score is not a negligible information. The achieved mean game scores at FruitCatcher and AnimalHurdler were higher at the end than at $1^{\text {st }}$ or $2^{\text {nd }}$ session indicating that participants' effort was still present. The mean score with HorseRunner was rather fluctuating. This information was not necessarily directly related to the improvement of balance abilities, but rather presented an intrinsic motivation factor for the participants (32).

\subsection{Conventional physiotherapy group}

The provided balance training program contributed to the statistically significant improvement of the two mobility tests, 10MWT and the more comprehensive test requiring standing up, the TUG. A detailed insight into the participant's data revealed that actually each individual patient improved his/her score in 
the applied clinical tests or measures were not sensitive due to the ceiling effect. With the exception of one patient who was not able to stand on his single limb with eyes closed and was also not able to finish the FSST. Closing the eyes deprived the participants of the visual information. Those with well-preserved proprioception and vestibular sensory system functionalities would have been compensating for vision. But the dysfunction of the vestibular system is often present in persons with stroke or brain injury and people suffering for middle cerebral artery stroke show large asymmetrical lateral displacement, responses to postural perturbation are asymmetrical and balance is hindered (33). Therefore in our case, balancing and postural control of the patients at baseline mainly relied on proprioceptive control. After the intervention statistically significant difference of means with the baseline was found using the ROM with eyes closed. Obviously the additional balance training had positive influence on the vestibular system functions, considering that the clinical instrument ROM is intended for vestibular disorders assessment (34) and the patients were deprived of visual information.

\subsection{Advantages of exergaming over conventional physiotherapy}

The additional exergaming was equivalent to the conventional physiotherapy in the control group in terms of clinical outcome. Both approaches demonstrated progress in ROM with closed eyes after the training. This means that no matter which of the additional balance training was added to the rehabilitation program, both would influence on the improvement of vestibular sensory system functionality.

The functional test FSST targeting vestibular disorders (20) evaluation also confirmed our findings. The positive changes in FSST could be related to the additional exergaming based balance training (U3 $=0.9)$ despite the short, but intensive program as in the control group we found minimal effect size (U3 $=0.4)$. Blenerhassett and Jayalath (35) reported that the main changes in FSST were noticed from the baseline to the second week and only minor changes were reported from week 2 to week 4 . The FSST was considered a dynamic balance test that could successfully assess changes during balance training in stroke population and provide ancillary information about daily living activities. Participant finishing the test in more than 12s were classified in a group with increased risk for falls (20). According to these criteria both participating groups were considered fallers before the additional balance training. However, only the exergaming group achieved the mean score of $10.24 \mathrm{~s}$, making the group for non-fallers. Besides, the change was considered statistically significant $(p=0.009)$. TUG and MWT both demonstrated clinically proven improvements, but no statistical difference between both approaches was found.

The major advantage of the exergaming approach was the access to the objective, measurable information like the COP. The COP area displacement was reported to be in correlation with the clinically proven tests for balance and posture evaluation (28) and furthermore we even found differences between the applied exergames. Therefore the appropriate choice of the exergame may help targeting specific balance or postural disorder.

The gaming group found the additional balance training challenging according to the achieved score which is in line with the findings of Hung et al (31) who reported on importance of game diversity and 
fun.

We have demonstrated that exergaming can be equivalent to conventional intensive balance training in the additional rehabilitation program. Furthermore, valuable results were demonstrated by FSST and tests with closed eyes. So far we do not expect that virtual reality based rehabilitation is far more effective than conventional rehabilitation (15), but in our opinion we could achieve promising results with the additional balance and mobility training without replacing any existing clinical method and possibly extend the rehabilitation period for a larger stroke population, if they can perform some exercises at home.

\subsection{Limitations of the study and future work}

The objective of the study was additional balance training in the rehabilitation program based on multiexergaming that may replace the additional balance training in patients recovering from stroke. We measured the kinematics of the whole body with the camera and COP with the low-cost force plate. The COP could be assessed also in the control group to make the results also objectively comparable, however the clinical exercises for balance training would have provided different, directly incomparable data. The solution might have been development of task specific tests that would be equal for both groups. Despite the games score was a good indicator of participants effort, we could have applied also the intrinsic motivation inventory (36) to both groups.

On the other hand the clinical tests demonstrated much higher variance than expected for the planned statistical power (0.8). The recruited sample size would have been large enough to cover the objective assessments, but unfortunately not all patients were eligible for the study. The small sample size caused violation of the assumption of sphericity (Mauchly's test) and statistical degrees of freedom were corrected in the study. We see a small sample size as a limiting factor to assure the sphericity of the data and also for decreasing the statistical power to the analysis of the clinical tests. We may suggest that the balance training was equivalent in both groups as reported by the literature (15) and rather medium to large effect sizes were found. But further randomized clinical trials are needed to promote exergaming. Particularly if the evaluation tool is intended for objective evaluation of the remote (tele)rehabilitation.

\section{Conclusions}

In the study advantages of the multi-exergames targeting balance, posture, single leg standing, weight shifting or simply just muscles strengthening by sit-to-stand exercise were demonstrated. There has been decades of scientific reporting that exergaming can contribute to the improvement of fine and gross motor skills in patients diagnosed with stroke. The contemporary approach provides non-negligible advantages over additional conventional balance training in terms of objective assessment and more targeted balance and posture disorders. .

Additionally the study showed that significant differences were found only in clinical tests closed eyes and FSST. This led us to the conclusions that both approaches could be equivalent in terms of 
effectiveness, however, the exergaming may require less strenuous work and physiotherapist's interventions and has a potential for telerehabilitation (37). We suggest that future studies should take into account also the motivation factor of the participants and examine whether the exergame difficulty level would affect the voluntary contribution of each individual and how such approach could also work out in home environment.

\section{Declarations}

Ethics approval and consent to participate

The study (Approval Number: 10112016) was approved by ethics committee of University Rehabilitation Institute, Republic of Slovenia and all participants provided an informed written consent. The procedure was in accordance with the principles of the Declaration of Helsinki on biomedical research on human beings, the provisions of Council of Europe Convention on the Protection of Human Rights and Dignity of the Human Being with regard to the Application of Biology and Medicine (Oviedo Convention) and the principles of Slovenian Code of medical ethics. The authors confirm that all ongoing and related trials for this drug/intervention are registered (ClinicalTrials.gov Identifier: NCT03282968)

Consent to publish

All authors, project group members and participant agreed on publication. Participant provided a written consent for the publication of photographs.

Availability of data and material

Anonymized data generated or analyzed during this study are included in this published article [and its supplementary information files]

Competing interests

The authors declare that they have no competing interests

Funding

REWIRE was partially funded by the European Commission under the 7th Framework Programme FP7ICT-2011 Call 7 - Personal Health System (ID 287713)

Slovenian Research Agency (research core funding No. P2-0228).

Authors' contributions

All authors read and approved the final manuscript.

Acknowledgements 
The authors would like to thank Nika Goljar, MD, PhD for medical advices. The authors also acknowledge the financial support from the Slovenian Research Agency (research core funding No. P2-0228).

\section{References}

1. Tyson SF, Hanley M, Chillala J, Selley A, Tallis RC. Balance disability after stroke. Phys Ther [Internet]. 2006 Jan [cited 2017 Dec 1];86(1):30-8. Available from: http://www.ncbi.nlm.nih.gov/pubmed/16386060

2. Kwakkel G. Impact of intensity of practice after stroke: issues for consideration. Disabil Rehabil [Internet]. 2009 Jan 7 [cited 2012 Jul 30];28(13-14):823-30. Available from: http://informahealthcare.com/doi/abs/10.1080/09638280500534861? prevSearch=allfield\%253A\%2528kwakkel\%2529\&searchHistoryKey=

3. Lang CE, MacDonald JR, Reisman DS, Boyd L, Jacobson Kimberley T, Schindler-Ivens SM, et al. Observation of Amounts of Movement Practice Provided During Stroke Rehabilitation. Arch Phys Med Rehabil [Internet]. 2009 Oct [cited 2017 Dec 1];90(10):1692-8. Available from: http://www.ncbi.nlm.nih.gov/pubmed/19801058

4. Foley NC, Teasell RW, Bhogal SK, Doherty T, Speechley MR. The efficacy of stroke rehabilitation: a qualitative review. Top Stroke Rehabil [Internet]. 2003 [cited 2017 Dec 1];10(2):1-18. Available from: http://www.ncbi.nlm.nih.gov/pubmed/13680515

5. Goršič M, Cikajlo I, Novak D. Competitive and cooperative arm rehabilitation games played by a patient and unimpaired person: Effects on motivation and exercise intensity. J Neuroeng Rehabil [Internet]. 2017 Dec 23 [cited 2017 Apr 11];14(1):submitted for publication. Available from: http://www.ncbi.nlm.nih.gov/pubmed/28330504

6. Oblak J, Cikajlo I, Matjacić Z. Universal haptic drive: a robot for arm and wrist rehabilitation. IEEE Trans Neural Syst Rehabil Eng [Internet]. 2010;18(3):293-302 ST-Universal haptic drive: a robot for. Available from: http://www.ncbi.nlm.nih.gov/pubmed/19846386

7. Krebs H, Ferraro M, Buerger SP, Newbery MJ, Makiyama A, Sandmann M, et al. Rehabilitation robotics: pilot trial of a spatial extension for MIT-Manus. J Neuroeng Rehabil [Internet]. 2004 Oct 26 [cited 2017 Dec 21];1(1):5. Available from: http://jneuroengrehab.biomedcentral.com/articles/10.1186/1743-0003-1-5

8. Dundar U, Toktas H, Solak O, Ulasli AM, Eroglu S. A Comparative Study of Conventional Physiotherapy Versus Robotic Training Combined with Physiotherapy in Patients with Stroke. Top Stroke Rehabil [Internet]. 2014 Nov 5 [cited 2017 Dec 21];21(6):453-61. Available from: http://www.ncbi.nlm.nih.gov/pubmed/25467393

9. Meuleman J, van Asseldonk E, van Oort G, Rietman H, van der Kooij H. LOPES II-Design and Evaluation of an Admittance Controlled Gait Training Robot With Shadow-Leg Approach. IEEE Trans Neural Syst Rehabil Eng [Internet]. 2016 Mar [cited 2017 Dec 21];24(3):352-63. Available from: http://www.ncbi.nlm.nih.gov/pubmed/26731771 
10. Chen I-C, Cheng P-T, Chen C-L, Chen S-C, Chung C-Y, Yeh T-H. Effects of balance training on hemiplegic stroke patients. Chang Gung Med J [Internet]. 2002 Sep [cited 2017 Dec 21];25(9):58390. Available from: http://www.ncbi.nlm.nih.gov/pubmed/12479619

11. Massion J. Movement, posture and equilibrium: interaction and coordination. Prog Neurobiol [Internet]. 1992 [cited 2017 Dec 1];38(1):35-56. Available from:

http://www.ncbi.nlm.nih.gov/pubmed/1736324

12. Langhorne $P$, Coupar F, Pollock A. Motor recovery after stroke: a systematic review. Lancet Neurol [Internet]. 2009 Aug [cited 2017 Dec 1];8(8):741-54. Available from:

http://www.ncbi.nlm.nih.gov/pubmed/19608100

13. Jack D, Boian R, Merians AS, Tremaine M, Burdea GC, Adamovich S V, et al. Virtual reality-enhanced stroke rehabilitation. IEEE Trans Neural Syst Rehabil Eng [Internet]. 2001;9:308-18 ST-Virtual reality-enhanced stroke rehab. Available from: http://www.ncbi.nlm.nih.gov/pubmed/11561668

14. Laver KE, Lange B, George S, Deutsch JE, Saposnik G, Crotty M. Virtual reality for stroke rehabilitation. Cochrane Database Syst Rev [Internet]. 2017 Nov 20 [cited 2017 Dec 1];11:CD008349. Available from: http://www.ncbi.nIm.nih.gov/pubmed/29156493

15. Corbetta D, Imeri F, Gatti R. Rehabilitation that incorporates virtual reality is more effective than standard rehabilitation for improving walking speed, balance and mobility after stroke: a systematic review. J Physiother [Internet]. 2015 Jul 1 [cited 2017 Dec 14];61(3):117-24. Available from: https://www.sciencedirect.com/science/article/pii/S1836955315000569

16. Pirovano M, Mainetti R, Lanzi PL, Borghese NA. Game Engines and Exergames to Guide Rehabilitation at Home. In Springer, Cham; 2014 [cited 2017 Dec 14]. p. 129-34. Available from: http://link.springer.com/10.1007/978-3-319-08072-7_25

17. Magill RA. Motor learning and control : concepts and applications / Richard A. Magill. - Version details - Trove [Internet]. 7th ed. Boston : McGraw-Hill; 2004 [cited 2017 Dec 4]. Available from: http://trove.nla.gov.au/work/19542376?selectedversion=NBD24624099

18. Leach J, Mancini M, Peterka R, Hayes T, Horak F. Validating and Calibrating the Nintendo Wii Balance Board to Derive Reliable Center of Pressure Measures. Sensors [Internet]. 2014 Sep 29 [cited 2017 Sep 1];14(10):18244-67. Available from: http://www.ncbi.nlm.nih.gov/pubmed/25268919

19. Moghadam M, Ashayeri H, Salavati M, Sarafzadeh J, Taghipoor KD, Saeedi A, et al. Reliability of center of pressure measures of postural stability in healthy older adults: Effects of postural task difficulty and cognitive load. Gait Posture [Internet]. 2011 Apr [cited 2017 Sep 1];33(4):651-5. Available from: http://www.ncbi.nlm.nih.gov/pubmed/21458272

20. Whitney SL, Marchetti GF, Morris LO, Sparto PJ. The reliability and validity of the Four Square Step Test for people with balance deficits secondary to a vestibular disorder. Arch Phys Med Rehabil [Internet]. 2007 Jan [cited 2012 Jul 14];88(1):99-104. Available from: http://www.ncbi.nlm.nih.gov/pubmed/17207683

21. Ng SS, Hui-Chan CW. The timed up \& go test: its reliability and association with lower-limb impairments and locomotor capacities in people with chronic stroke. Arch Phys Med Rehabil 
[Internet]. 2005 Aug [cited 2011 Sep 19];86(8):1641-7. Available from:

http://www.ncbi.nlm.nih.gov/pubmed/16084820

22. Nagano $\mathrm{K}$, Hori $\mathrm{H}$, Muramatsu K. A comparison of at-home walking and 10-meter walking test parameters of individuals with post-stroke hemiparesis. J Phys Ther Sci [Internet]. 2015 Feb [cited 2017 Dec 1];27(2):357-9. Available from: http://www.ncbi.nlm.nih.gov/pubmed/25729167

23. Longridge NS, Mallinson Al. Clinical Romberg Testing Does Not Detect Vestibular Disease. Otol Neurotol [Internet]. 2010 Jul [cited 2017 Dec 1];31(5):803-6. Available from: http://www.ncbi.nlm.nih.gov/pubmed/20502374

24. Horn LB, Rice T, Stoskus JL, Lambert KH, Dannenbaum E, Scherer MR. Measurement Characteristics and Clinical Utility of the Clinical Test of Sensory Interaction on Balance (CTSIB) and Modified CTSIB in Individuals With Vestibular Dysfunction. Arch Phys Med Rehabil [Internet]. 2015 Sep [cited 2017 Dec 1];96(9):1747-8. Available from: http://www.ncbi.nlm.nih.gov/pubmed/26550644

25. Cohen J. Statistical power analysis for the behavioral sciences [Internet]. Academic Press; 1977 [cited 2018 Jan 4]. 474 p. Available from:

https://www.sciencedirect.com/science/book/9780121790608

26. Hentschke H, Stüttgen MC. Computation of measures of effect size for neuroscience data sets. Eur J Neurosci [Internet]. 2011 Dec [cited 2019 Apr 2];34(12):1887-94. Available from: http://www.ncbi.nlm.nih.gov/pubmed/22082031

27. Mauchly JW. Significance Test for Sphericity of a Normal \$n\$-Variate Distribution. Ann Math Stat [Internet]. 1940 Jun [cited 2017 Dec 13];11(2):204-9. Available from: http://projecteuclid.org/euclid.aoms/1177731915

28. Lopes PG, Lopes JAF, Brito CM, Alfieri FM, Rizzo Battistella L. Relationships of Balance, Gait Performance, and Functional Outcome in Chronic Stroke Patients: A Comparison of Left and Right Lesions. Biomed Res Int [Internet]. 2015 [cited 2017 Dec 13];2015:716042. Available from: http://www.ncbi.nlm.nih.gov/pubmed/26583129

29. Paillex R, So A. Changes in the standing posture of stroke patients during rehabilitation. Gait Posture [Internet]. 2005 Jun [cited 2017 Dec 13];21(4):403-9. Available from: http://www.ncbi.nlm.nih.gov/pubmed/15886130

30. Rode G, Tiliket C, Boisson D. Predominance of postural imbalance in left hemiparetic patients. Scand J Rehabil Med [Internet]. 1997 Mar [cited 2017 Dec 13];29(1):11-6. Available from: http://www.ncbi.nlm.nih.gov/pubmed/9084100

31. Hung Y-X, Huang P-C, Chen K-T, Chu W-C. What Do Stroke Patients Look for in Game-Based Rehabilitation: A Survey Study. Medicine (Baltimore) [Internet]. 2016 Mar [cited 2017 Dec 14];95(11):e3032. Available from: http://www.ncbi.nlm.nih.gov/pubmed/26986120

32. Goršič M, Cikajlo I, Novak D. Motivation and exercise intensity in competition and cooperation between a patient and unimpaired person in arm rehabilitation. In: Proceedings of the 2016 International Conference on NeuroRehabilitation. Segovia, Spain; 2016. 
33. Marsden JF, Playford DE, Day BL. The vestibular control of balance after stroke. J Neurol Neurosurg Psychiatry [Internet]. 2005 May [cited 2017 Dec 15];76(5):670-8. Available from: http://www.ncbi.nlm.nih.gov/pubmed/15834025

34. Gottshall K. Vestibular rehabilitation after mild traumatic brain injury with vestibular pathology. NeuroRehabilitation [Internet]. 2011 [cited 2017 Dec 15];29(2):167-71. Available from: http://www.ncbi.nlm.nih.gov/pubmed/22027078

35. Blennerhassett JM, Jayalath VM. The Four Square Step Test is a Feasible and Valid Clinical Test of Dynamic Standing Balance for Use in Ambulant People Poststroke. Arch Phys Med Rehabil [Internet]. 2008 Nov [cited 2017 Dec 18];89(11):2156-61. Available from:

http://www.ncbi.nlm.nih.gov/pubmed/18996245

36. Goršič M, Cikajlo I, Novak D. Motivation and exercise intensity in competition and cooperation between a patient and unimpaired person in arm rehabilitation. Vol. 15, Biosystems and Biorobotics. 2017.

37. Cikajlo I, Rudolf M, Goljar N, Burger H, Matjačić Z. Telerehabilitation using virtual reality task can improve balance in patients with stroke. Disabil Rehabil. 2012;

\section{Tables}

Table 1. Mean COP area during exergaming with 3 different games (Fruit Catcher, Horse rider and Animal hurdler) for 5 consecutive sessions. Smaller area of the COP reflects to smaller motion range - in these balance games means improved balance.

\begin{tabular}{|l|c|c|c|c|c|}
\hline & \multicolumn{5}{|c|}{ COP area (mean \pm standard deviation) } \\
\hline & \multicolumn{5}{|c|}{ Session number } \\
\hline Exergame & 1 & 2 & 3 & 4 & 5 \\
\hline Fruit catcher & $23.84 \pm 7.43^{*}$ & $43.91 \pm 28.93$ & $60.11 \pm 34.95$ & $48.59 \pm 32.57$ & $40.06 \pm 21.95$ \\
\hline Horse runner & $24.34 \pm 6.82$ & $23.33 \pm 7.42$ & $18.70 \pm 9.71$ & $16.71 \pm 11.03$ & $20.56 \pm 8.70$ \\
\hline Animal hurdler & $128.77 \pm 66.27$ & $102.54 \pm 37.16$ & $117.51 \pm 39.78$ & $90.03 \pm 21.26$ & $107.91 \pm 31.66$ \\
\hline
\end{tabular}

Table 2. Results of the clinical tests in exergaming group before and after the additional balance training . 


\begin{tabular}{|c|c|c|c|c|c|c|c|c|c|}
\hline \multirow[b]{3}{*}{ Test } & \multicolumn{7}{|c|}{ gaming group } & \multirow{3}{*}{\begin{tabular}{|c|} 
Cohen's \\
U3
\end{tabular}} & \multirow{3}{*}{$\frac{\text { U3 CI }}{[\min \max }$} \\
\hline & \multicolumn{2}{|c|}{ before } & \multicolumn{2}{|c|}{ after } & \multirow{2}{*}{$\begin{array}{c}\text { Bartlett test } \\
\mathrm{c}^{2}(\mathrm{p} \text {-value }) \\
\end{array}$} & \multirow{2}{*}{$\begin{array}{c}\text { Wilcoxon } \\
\text { p-value } \\
\end{array}$} & \multirow{2}{*}{\begin{tabular}{|l|} 
ANOVA \\
$p$-value \\
\end{tabular}} & & \\
\hline & mean & $\mathrm{SD}$ & mean & SD & & & & & \\
\hline FSST & 13.21 & 3.90 & 10.24 & 2.44 & $27.786(0.000)$ & $0.009 *$ & & 0.9 & {$\left[\begin{array}{ll}0.3 & 1\end{array}\right]$} \\
\hline TUG & 9.56 & 2.69 & 8.46 & 2.02 & $25.187(0.000)$ & 0.092 & & 0.7 & {$\left[\begin{array}{lll}0.2 & 1\end{array}\right]$} \\
\hline $10 \mathrm{MWT}$ & 8.76 & 2.48 & 7.14 & 1.60 & $23.689(0.000)$ & 0.008* & & 0.9 & {$\left[\begin{array}{ll}0.3 & 1\end{array}\right]$} \\
\hline CTSIB eyes open & 38.83 & 13.15 & 43.52 & 4.68 & $\operatorname{Inf}(0.000)$ & 0.18 & & 0.5 & {$\left[\begin{array}{ll}0.1 & 0.5\end{array}\right]$} \\
\hline CTSIB eyes closed & 31.39 & 19.14 & 36.69 & 17.52 & $0.157(0.984)$ & & 0.341 & 0.5 & {$\left[\begin{array}{ll}0 & 0.5\end{array}\right]$} \\
\hline STOLL eyes open & 18.21 & 19.30 & 23.74 & 19.01 & $0.062(0.996)$ & & 0.575 & 0.2 & {$\left[\begin{array}{ll}0 & 0.85\end{array}\right]$} \\
\hline STOLL eyes closed & 1.61 & 1.18 & \begin{tabular}{|l|}
4.62 \\
\end{tabular} & 5.01 & $14.122(0.003)$ & 0.093 & & 0.4 & {$\left[\begin{array}{ll}0.1 & 0.7\end{array}\right]$} \\
\hline STORL eyes open & 21.53 & 20.42 & 23.87 & 19.40 & $0.466(0.926)$ & & 0.482 & 0.4 & {$\left[\begin{array}{ll}0 & 0.9\end{array}\right]$} \\
\hline STORL eyes closed & 3.92 & 7.09 & 2.82 & 2.02 & $29.627(0.00002)$ & 0.674 & & 0.5 & {$\left[\begin{array}{ll}0 & 0.85\end{array}\right]$} \\
\hline ROM eyes open & 44.33 & 2.12 & 43.75 & 3.95 & Inf $(0.000)$ & 0.317 & & 0.5 & {$\left[\begin{array}{ll}0.5 & 0.5\end{array}\right]$} \\
\hline ROM eyes closed & 36.79 & 13.66 & 41.49 & 11.10 & $5.108(0.164)$ & & $0.046 *$ & 0.5 & {$\left[\begin{array}{ll}0 & 0.5\end{array}\right]$} \\
\hline sROM eyes open & 34.56 & 17.02 & 42.11 & 8.46 & $6.536(0.088)$ & & 0.369 & 0.5 & {$\left[\begin{array}{ll}0 & 0.75\end{array}\right]$} \\
\hline sROM eyes closed & 19.65 & 19.16 & 18.96 & 19.10 & $2.147(0.542)$ & & 0.969 & 0.6 & {$\left[\begin{array}{ll}0.1 & 0.9\end{array}\right]$} \\
\hline
\end{tabular}

$* p<0.05$ statistical significant difference

Table 3. Results of the clinical tests in control group before and after the additional balance training.

\begin{tabular}{|c|c|c|c|c|c|c|c|c|c|}
\hline & \multicolumn{7}{|c|}{ Conventional physiotherapy - control group } & \multirow{3}{*}{\begin{tabular}{|c|} 
Cohen-s \\
U3 \\
\end{tabular}} & \multirow{3}{*}{$\frac{\text { U3 CI }}{[\text { min max }}$} \\
\hline & \multicolumn{2}{|c|}{ before } & \multicolumn{2}{|c|}{ after } & \multirow{2}{*}{$\begin{array}{c}\text { Bartlett test } \\
\mathrm{p} \text {-value } \\
\end{array}$} & \multirow{2}{*}{$\begin{array}{c}\text { Wilcoxon } \\
\text { p-value } \\
\end{array}$} & \multirow{2}{*}{\begin{tabular}{|l|} 
ANOVA \\
$p$-value \\
\end{tabular}} & & \\
\hline Test & mean & $\mathrm{SD}$ & mean & $\mathrm{SD}$ & & & & & \\
\hline FSST & 12.75 & 12.10 & 14.50 & 13.78 & $27.786(0.00004)$ & 0.575 & & 0.4 & {$\left[\begin{array}{ll}0 & 0.9\end{array}\right]$} \\
\hline TUG & 15.18 & 10.20 & 12.17 & 6.74 & $25.187(0.00001)$ & $0.011 *$ & & 0.6 & {$\left[\begin{array}{ll}0.2 & 1\end{array}\right]$} \\
\hline $10 \mathrm{MWT}$ & 12.34 & 8.22 & 9.82 & 5.68 & $23.689(0.00003)$ & $0.008 *$ & & 0.6 & {$\left[\begin{array}{lll}0.2 & 1\end{array}\right]$} \\
\hline CTSIB eyes open & 43.50 & 4.74 & 45.00 & 0.00 & $\operatorname{Inf}(0.000)$ & 0.317 & & 0.5 & {$\left[\begin{array}{lll}0.5 & 0.5\end{array}\right]$} \\
\hline CTSIB eyes closed & 29.13 & 18.16 & 34.77 & 16.81 & $0.157(0.984)$ & & 0.341 & 0.3 & {$\left[\begin{array}{ll}0 & 0.75\end{array}\right]$} \\
\hline STOLL eyes open & 18.82 & 19.83 & 20.33 & 20.57 & $0.062(0.995)$ & & 0.575 & 0.5 & {$\left[\begin{array}{ll}0.1 & 0.8\end{array}\right]$} \\
\hline STOLL eyes closed & 2.20 & 3.16 & 2.35 & 3.40 & $14.122(0.003)$ & 0.273 & & 0.5 & {$\left[\begin{array}{lll}0.15 & 0.9\end{array}\right]$} \\
\hline STORL eyes open & 13.31 & 16.36 & 19.33 & 18.07 & $0.466(0.926)$ & & 0.482 & 0.4 & {$\left[\begin{array}{lll}0.05 & 0.8\end{array}\right]$} \\
\hline STORL eyes closed & 1.29 & 2.27 & 1.16 & 1.29 & $29.627(0.00002)$ & 0.753 & & 0.2 & {$\left[\begin{array}{lll}0.05 & 0.9\end{array}\right]$} \\
\hline ROM eyes open & 45.00 & 0.00 & 45.00 & 0.00 & $\operatorname{Inf}(0.000)$ & - & & 0.5 & {$\left[\begin{array}{lll}0.5 & 0.5\end{array}\right]$} \\
\hline ROM eyes closed & 26.46 & 19.85 & 40.29 & 9.94 & $5.108(0.164)$ & & $0.046 *$ & 0.2 & {$\left[\begin{array}{lll}0 & 0.5\end{array}\right]$} \\
\hline sROM eyes open & 31.90 & 21.12 & 34.03 & 18.07 & $6.536(0.088)$ & & 0.369 & 0.5 & {$\left[\begin{array}{ll}0.1 & 0.8\end{array}\right]$} \\
\hline sROM eyes closed & 8.57 & 13.36 & 9.67 & 13.42 & $2.147(0.542)$ & & 0.969 & 0.4 & {$\left[\begin{array}{lll}0.05 & 0.9\end{array}\right]$} \\
\hline
\end{tabular}

${ }^{*} \mathrm{p}<0.05$ statistical significant difference, A - ANOVA

Table 4. Analyzing differences between the exergaming group and the conventional physiotherapy control group. 


\begin{tabular}{|l|c|c|c|c|}
\hline \multicolumn{1}{|c|}{ variable } & $\begin{array}{c}\text { control } \\
\text { group }\end{array}$ & $\begin{array}{c}\text { gaming } \\
\text { group }\end{array}$ & $\begin{array}{c}\text { Levene's Test } \\
(\mathrm{F} / \mathrm{p})\end{array}$ & $\begin{array}{c}\text { t-test Equality of Means (p- } \\
\text { value) }\end{array}$ \\
\hline Gender (M/F) & $9 / 1$ & $6 / 4$ & $12.05 / 0.03$ & 0.138 \\
\hline Age (mean/SD) & $51.8 / 15.48$ & $50.3 / 7.90$ & $2.19 / 0.156$ & 0.788 \\
\hline $\begin{array}{l}\text { Affected side } \\
\text { (L/R/both) }\end{array}$ & $4 / 6 / 0$ & $5 / 4 / 1$ & $1.45 / 0.245$ & 1 \\
\hline MMSE & 27.6 & 28.6 & $1.33 / 0.263$ & 0.200 \\
\hline Stroke (years) & 7.4 & 4 & $5.16 / 0.036$ & 0.235 \\
\hline
\end{tabular}

\section{Figures}




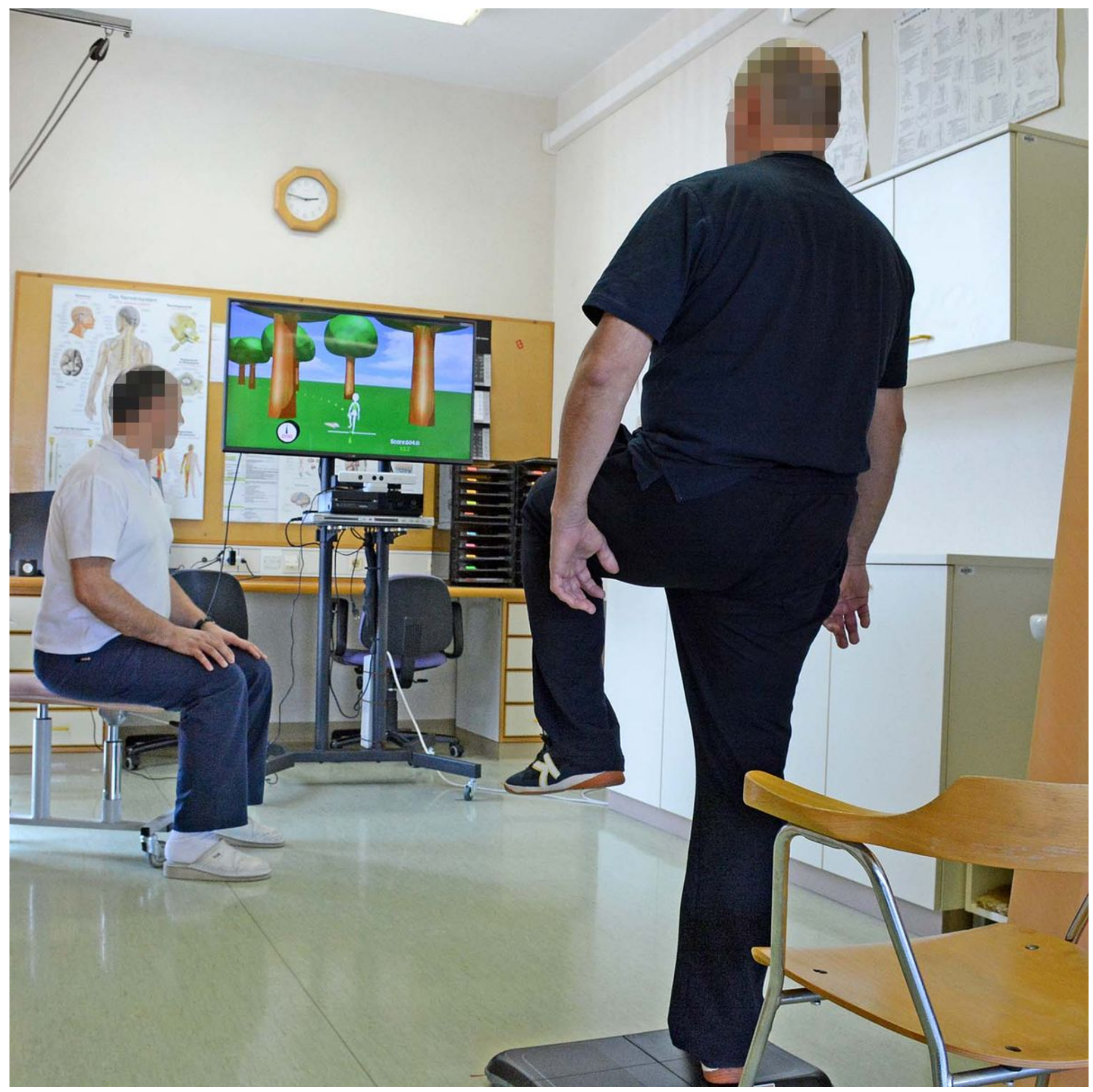

\section{Figure 1}

Setup for the exergaming participants. The participants were standing on the Wii Balance board and played 3 different games. MS Kinect camera tracked the participants' movements. 

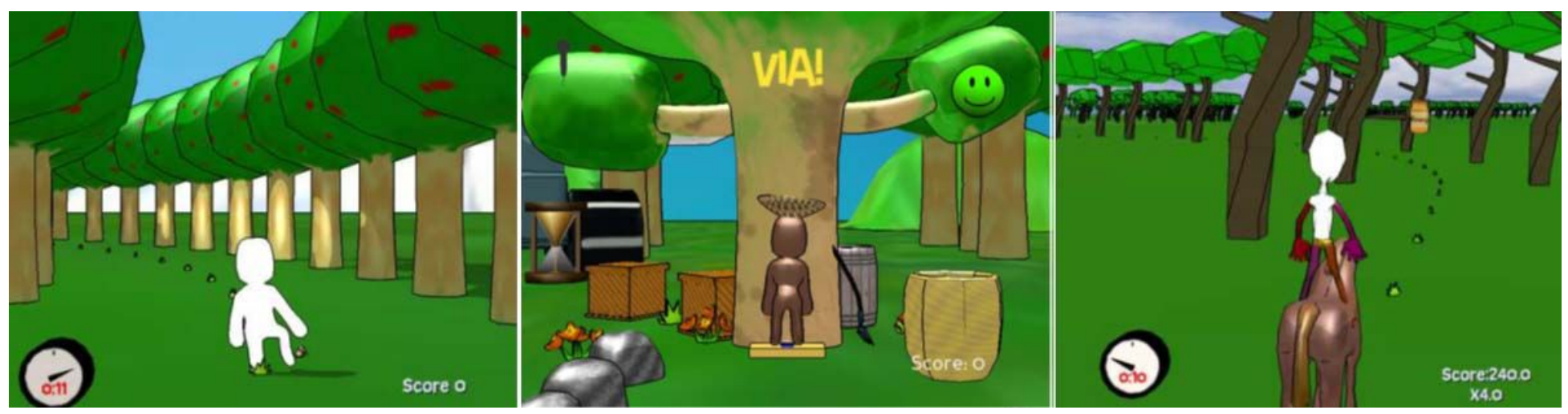

Figure 2

All games were intended for balance and posture, but Animal Hurdler game (1) also for weight shift and single leg standing, Fruit Catcher game (2) also for weight shift and steps and The Horse Runner game (3) also for sit-to-stand exercise. 


\section{CONSORT Flow Diagram}

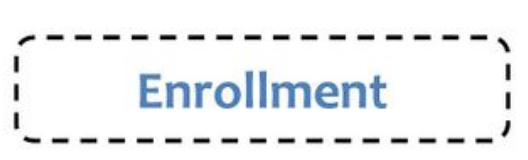

Assessed for eligibility $(n=30)$

Included $(\mathrm{n}=20)$

- Not meeting inclusion criteria $(n=7)$

- Other reasons $(n=3)$

Allocated to intervention $(n=20)$

- Received allocated intervention $(n=20)$
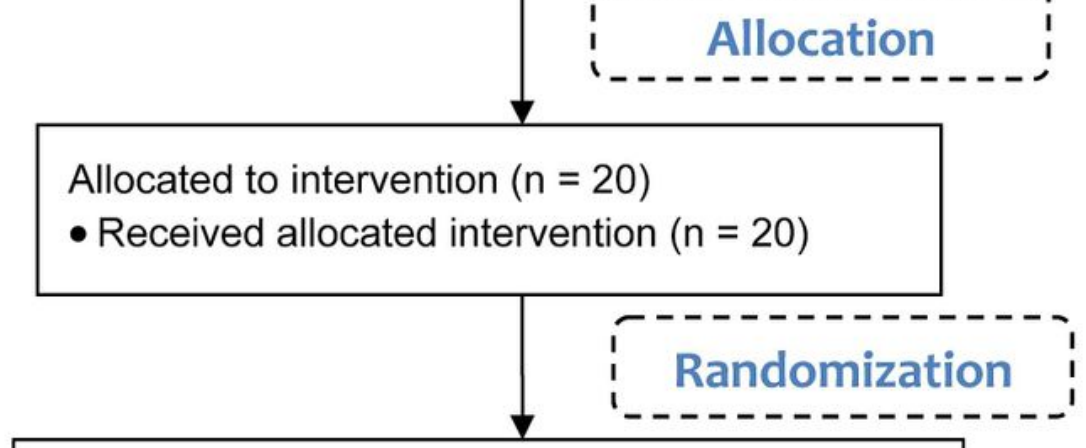

(A) Conventional physiotherapy group $(n=10)$

(B) Exergaming group $(n=10)$

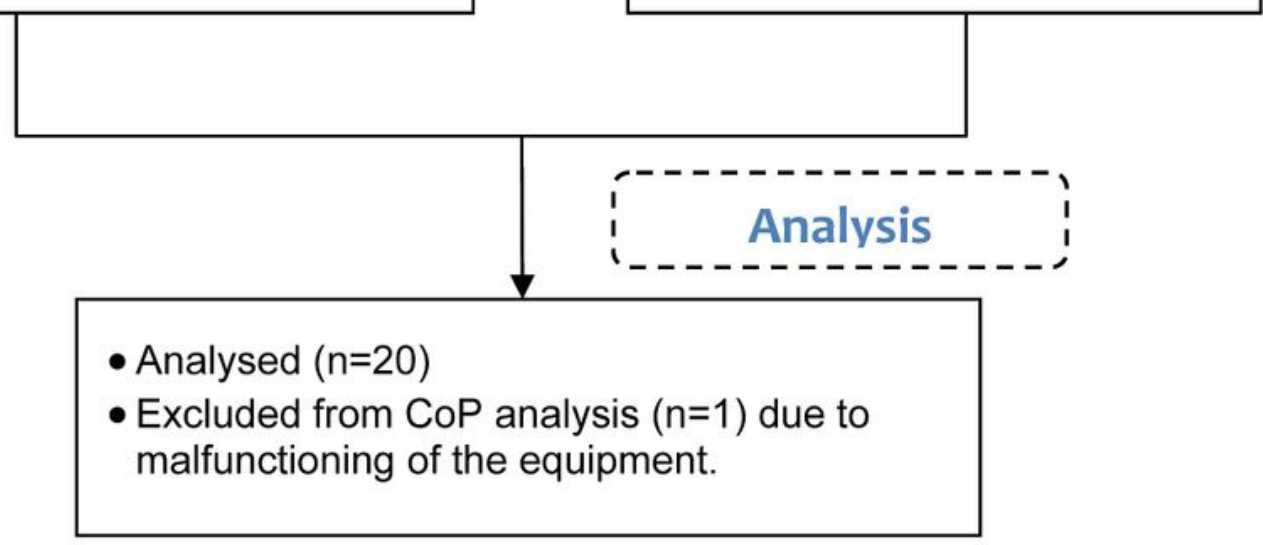

Figure 3

CONSORT Flow Diagram 


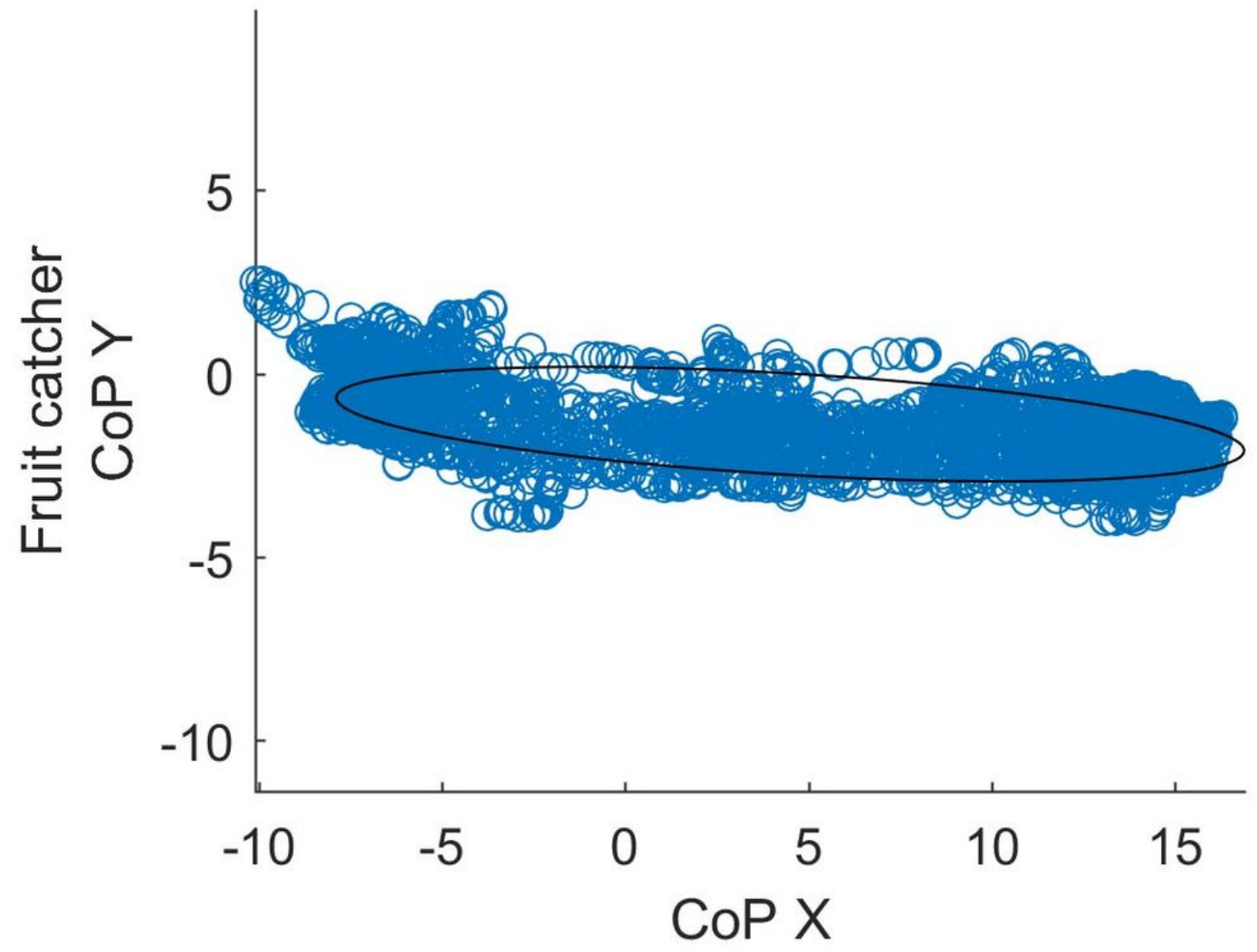

Figure 4

The $95 \%$ of the area covered by the ellipse was determined by calculating the eigenvectors. 


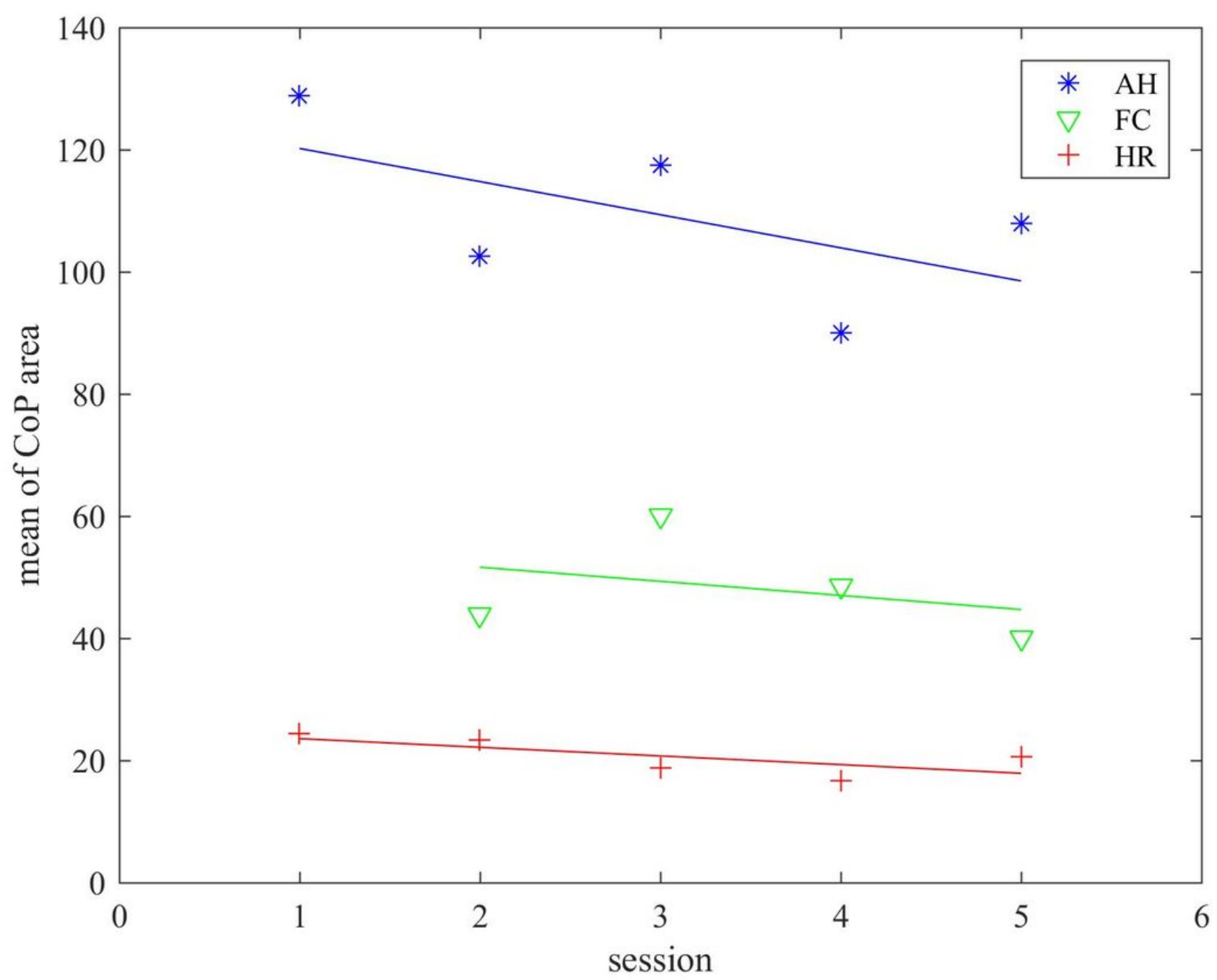

Figure 5

Regression lines of COP area over the sessions demonstrated that there had been an improvement of balance capabilities in all three applied exergames. However, there was no statistically significant difference between the Fruit catcher (FC) and Horse runner (HR) games in terms of COP area $(p=0.088)$, but only between the Animal hurdler $(\mathrm{AH})$ and other two $(\mathrm{p}<0.003)$. 

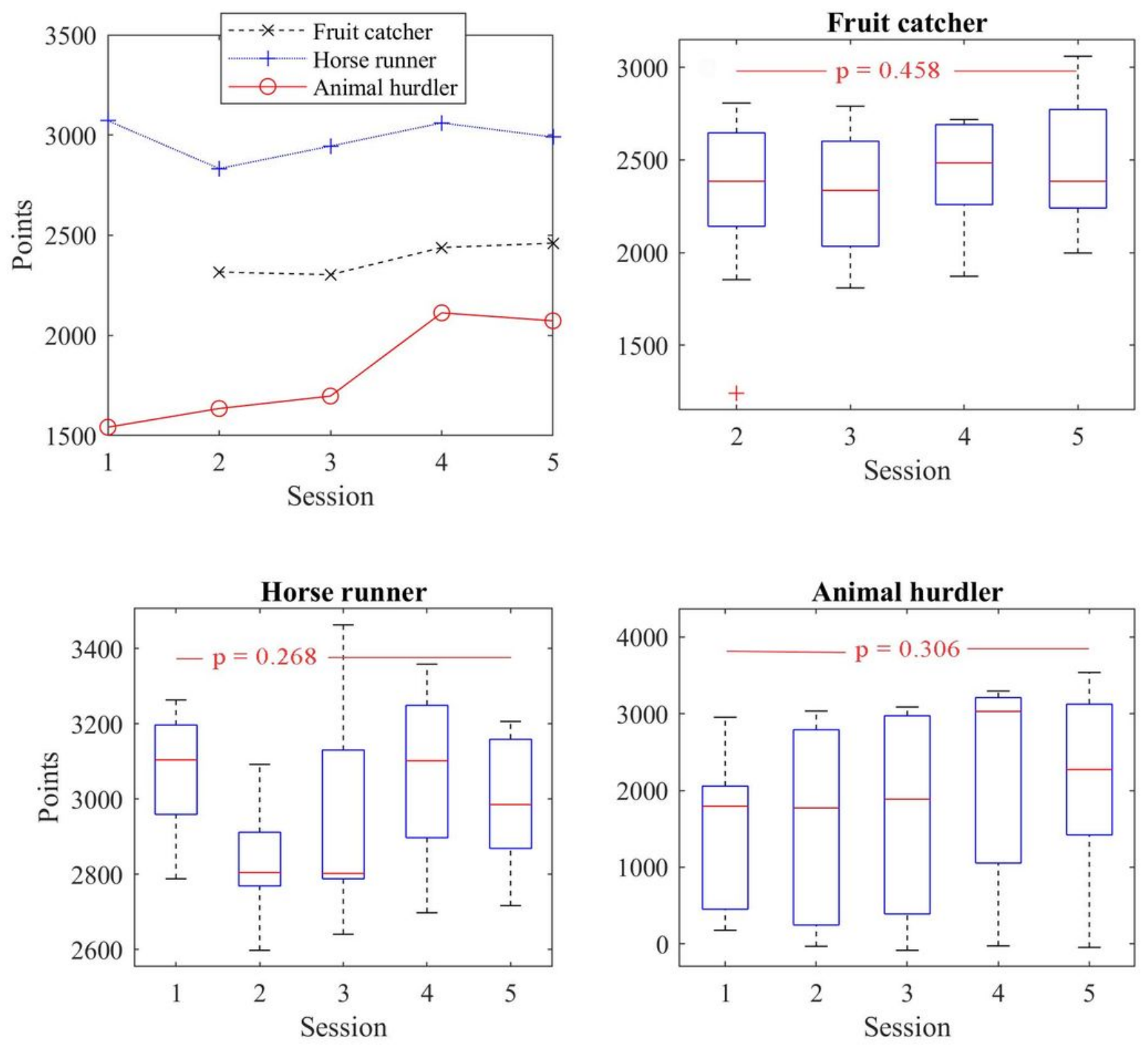

Figure 6

Points achieved in each exergame FruitCatcher (FC), AnimalHurdler (AH) and Horse Runner (HR). Mean values show that participants performed better at the last and pre-last sessions with the $\mathrm{FC}$ and $\mathrm{AH}$ games. However, the median values and the interquartile ranges revealed that despite of the noticeable progress in $\mathrm{AH}$ we had many participants with low score $(p>0.05)$. 

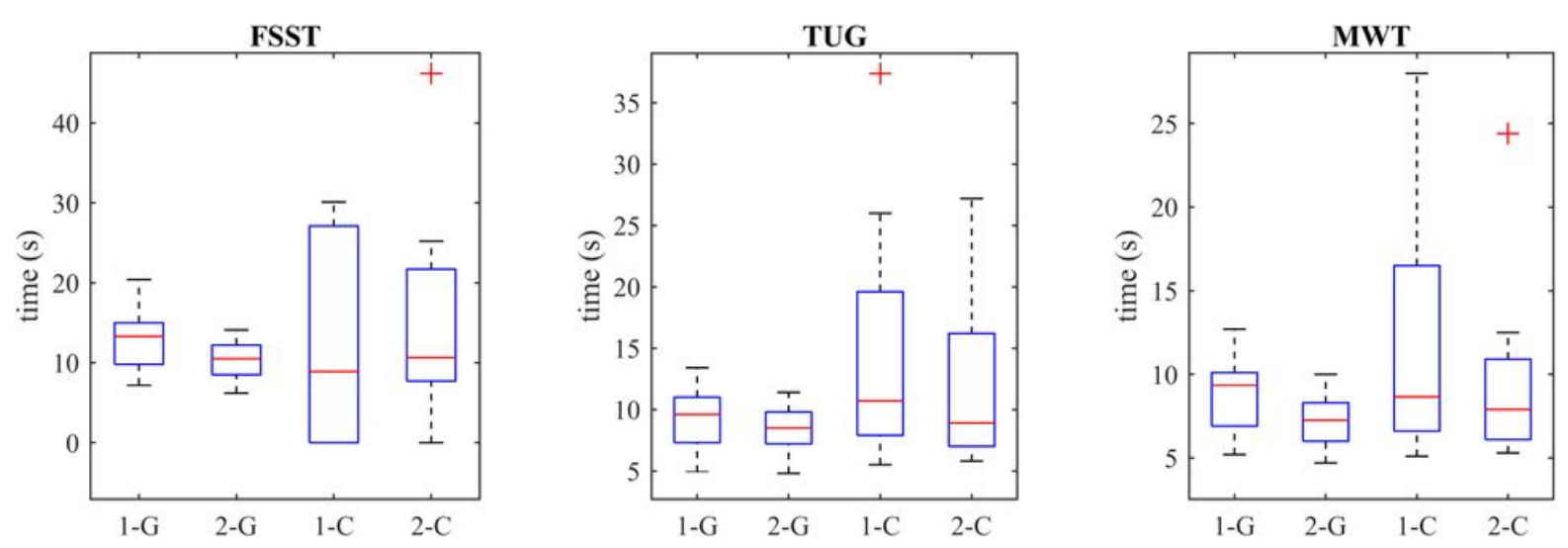

\section{Figure 7}

The gaming group $(G)$ demonstrated functional improvements after the training (2-G) in FSST ( $p=$ $0.009)$, TUG $(p=0.092)$ and MWT $(p=0.008)$ tests. The control group (C) also improved their TUG and MWT time comparing to the initial results (1-C). Substantive differences were found between the groups in MWT $(\mathrm{U} 3=0.7)$
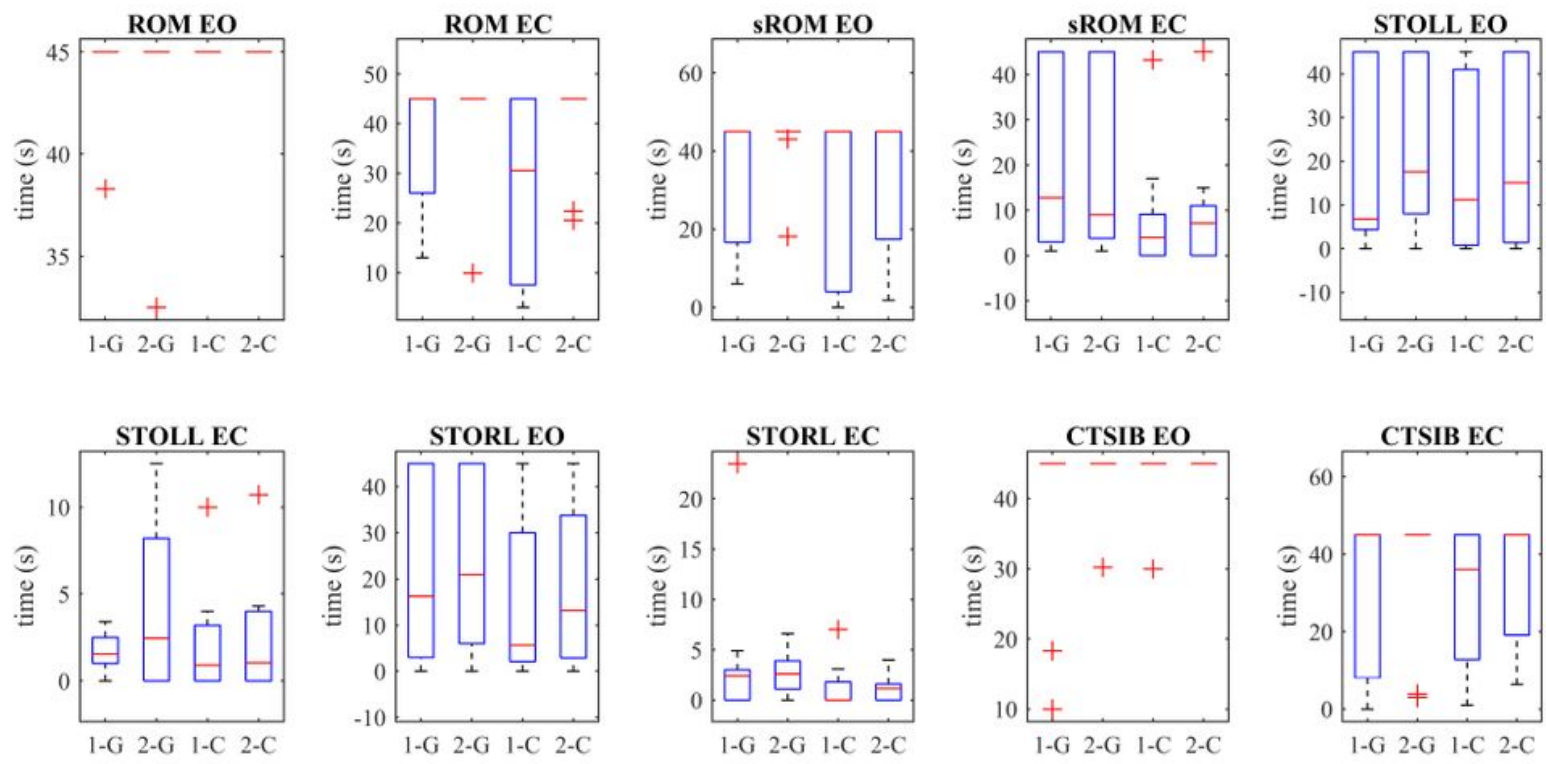

\section{Figure 8}

The clinical tests ROM, STOLL, STORL and CTSIB showed improvements of median score after the training (2), except the sROM with closed eyes for the gaming group $(G)$. However, differences between the control (C) and gaming $(G)$ groups were found only in two tests; STORL EC ( $p=0.035$, U3 $=0.3)$ and $\operatorname{sROM~EC~}(p=0.05, \mathrm{U} 3=0.4)$.

\section{Supplementary Files}

This is a list of supplementary files associated with this preprint. Click to download. 
- supplement1.pdf

- supplement2.png

- supplement2.png

- supplement4.png 